\title{
Endodontic Imaging as an Aid to Forensic Personal Identification
}

\author{
Running Title: \\ Endodontic Imaging in Forensic Identification
}

Alexander Stewart Forrest and Henry Yuan-Heng Wu

Address at which this work was carried out:

Forensic Pathology Unit

Queensland Health Forensic and Scientific Services,

39 Kessels Road,

Coopers Plains, 4109

Authors:

Alexander Stewart Forrest

Associate Professor

School of Biomolecular and Physical Sciences,

Griffith University

170 Kessels Rd

Nathan, Queensland 4111

Tel: +61737357546

Fax: +61 737355303

a.forrest@griffith.edu.au

and

Forensic Odontologist

Queensland Health Forensic and Scientific Services, 39 Kessels Road,

Coopers Plains, 4109

Tel: +61730009388

Fax: +61 732749201

Henry Yuan-Heng Wu

Forensic Odontologist

Queensland Health Forensic and Scientific Services, 39 Kessels Road,

Coopers Plains, 4109

Tel: +61 730009388

Fax: +61 732749201

Henry_Wu@health.qld.gov.au

\section{Address for Correspondence:}

Associate Professor Alex Forrest

School of Biomolecular and Physical Sciences,

Griffith University Nathan Campus

170 Kessels Rd 
Nathan, Queensland 4111

Tel: +61 737357546

Fax: +61 737355303

a.forrest@griffith.edu.au 


\section{Abstract}

Identification by dental comparison between records of a missing person and the dentition of a deceased individual depends on recognition of concordant features common to both with no unexplained discrepancies. While written dental records are commonly used for this task, we believe they should not be the preferred basis of comparison because they do not derive directly from an individual and are potentially prone to errors, inaccuracies and misinterpretation.

Images, however, are a direct representation of a physical item, and are an objective method of recording information. Radiographs are images that capture the unique morphological features of teeth, surrounding structures, and physical detail of past dental treatment resulting in changes to a dentition.

Taking post-mortem radiographs in such a way as to duplicate as closely as possible the conditions under which the ante-mortem radiographs were secured permits demonstrably rigorous methods of comparison, raising the probative value of the outcome. In this context post-treatment endodontic radiographs present a particularly rich source of features on which individuation can be achieved, especially considering that alteration of endodontic restorations is less frequently undertaken than is common on intra-coronal restorations. We illustrate various techniques with a series of cases and discuss the parameters for success.

\section{Keywords:}

Dental Records, Endodontics, Forensic Dentistry, Human Identification, Radiography 


\section{Introduction}

In coronial investigations, identification of a deceased individual plays a major role because a death certificate and related documentation cannot be issued until a Coroner is satisfied as to their identity. Most often this will not be controversial, and visual identification is commonly accomplished by family, friends or colleagues of the deceased. However there are situations in which this is not advisable, and some in which it is impossible, due to traumatic injury, incineration, decomposition or other factors.

In such cases, expert examination may be required to compare features of the deceased with records obtained for a missing individual. Depending on the situation, DNA, fingerprints and forensic odontology can all provide such advice and this will generally be accepted on its merits without further qualification if the basis for the comparison is clear and unequivocal. These three groups are therefore known collectively as "major identifiers".

In this context, the task of the forensic odontologist is to compare the dental records of a suspected or known missing person with the features of the dentition and oral structures of the deceased to determine the degree of correspondence, and to provide an expert opinion embodying the findings to the identifying authority, who has the final responsibility for identification (1). In Australia, this is the Coroner.

Written dental records comprise documents which attempt to convey in written language, and often graphically by means of an odontogram, features observed during dental examination, and details of dental procedures performed on a patient. They may not be written contemporaneously. They may indeed be written by an individual different from the operator, for example, a dental assistant, for reasons of infection control. They are therefore prone to errors of transcription, recall and interpretation, and in the worst instance, may record what a person thinks they heard someone say they thought they saw or did.

Because of this propensity for error, and without an objective source against which to compare this written record, such errors may not be detectable. In the forensic context, such errors may lead to failure to identify individuals correctly unless the forensic expert is aware of this issue and provides an appropriately conservative opinion. In some cases, even when no such errors exist, insufficient detail is recorded in the written record to allow a person to be identified on the basis of that information alone.

Radiographs are, however, images displaying details of the physical features of the item recorded. In the forensic context, they form objective records of a person and derive directly from that person; they are not surrogate records in the way that written records are, they record morphological details of everything in their field of view, and are not prone to the potential errors inherent in a purely written document. Importantly, they can be accurately duplicated by a different operator at a different time on the same patient. They are therefore extremely useful in the comparison process for personal forensic identification. 
Clearly, the more detailed and distinctive the morphology recorded in an image, the better the basis for comparison with a similar radiograph of an unknown person to establish that both images derive from the same individual. Dental treatment tends to leave radiographic evidence with detailed morphology that is likely to be unique, and therefore has high probative value in such a comparison. In this context, radiographs of endodontic treatments are an excellent source of individuating features based on their detailed distinctive morphology.

\section{Materials and Methods}

Due to their relative rarity and their unique expression of individual morphological features, root canal treatments are a very fruitful source of features that can be used to differentiate between dentitions and, by extension, demonstrate similarity to a high degree for identification by image comparison. Furthermore, root canal procedures are commonly radiographed prior to, during, at the end of, and periodically following treatment to provide a record of the outcome. Such post-treatment radiographs are therefore commonly available as part of the patient's dental record, and objectively document the specific morphological detail of the restoration and surrounding teeth and radiopaque supporting structures.

The purpose of the comparison process is to establish that these radiographs originate either from the same person (identification) or from different people (exclusion).

The success of the technique is dependent on the similarity of the parameters with which the two comparison images (ante-mortem and post-mortem) have been taken. Examination of the ante-mortem radiograph reveals information about the film/sensor position, the tube position, the image exposure, and the relative magnification or distortion of the image as taken in the dental surgery. All of these parameters need to be reproduced as accurately as possible in the post-mortem radiograph in order to render the two images directly comparable. Case 1 (below) illustrates the effect of not duplicating the x-ray tube and sensor position correctly in a post-mortem case.

Achieving this degree of accuracy in image reproduction commonly necessitates taking a number of post-mortem images in order to obtain a satisfactory result. Initial inspection of the first radiograph usually allows assessment as to whether the morphology of the root treatment, filling and dental morphology are sufficiently similar that exclusion can be determined at that stage. If not, then subsequent radiographs are taken to progressively improve image comparability until a satisfactory result is achieved.

The difficulties inherent in achieving such excellence in post-mortem radiography should not be underestimated. Trauma can cause substantial changes in body shape and position, and this can render the placement of a radiographic sensor or x-ray tube extremely difficult. Incinerated persons commonly undergo severe muscle contraction that bends limbs into severely flexed positions. Such bent limbs may restrict access for an x-ray tube. Further, incinerated teeth and jaws are frequently very fragile, and attempts to dissect jaws or teeth can cause loss of remaining tooth structure, compromising the identification outcome. One of the authors (ASF) has found that use of a hand held $\mathrm{x}$-ray generating device can make this process much simpler and 
may significantly enhance the quality of the outcome. Such devices are now commonly used by forensic odontologists in Australian mortuaries and in mass disaster scenarios.

A further difficulty is that the post-mortem radiographs have to accurately reproduce errors in technique that may be present in the supplied patient radiographs. This can be extremely challenging, and an excellent knowledge of radiography is required to identify those errors and to recreate them. The goal is not therefore to produce perfect radiographs of the deceased, but to simulate those provided in the patient record.

Once comparable post-mortem and ante-mortem images have been achieved, they can then be compared to determine the probability that they recorded images from the same individual, forming the objective basis for the expert opinion to be provided to the coroner.

The comparison process may comprise visual comparison (Cases 1 and 2) or the radiographs may be sufficiently similar that image superimposition can be achieved (Case 3). Ideally, subtraction imaging is used to demonstrate the concordances between the two images (Case 4) if the ante-mortem and post-mortem radiographs show the requisite level of similarity. In all cases, the requirement is to demonstrate the objective basis for the opinion, satisfying the Basis Rule for Expert Evidence to support legal processes.

\section{Results}

Four cases were selected from the records of the Forensic Odontology service at Queensland Health Forensic and Scientific Services (QHFSS) which used comparison of endodontic restorations as part of the identification process. These were deidentified and are shown as cases $1-4$ below:

\section{Case 1}

\section{Figure 1}

The missing person radiograph is on the left, and the image of the deceased is on the right. A brief hand-written note and a dental radiograph were supplied as the missing person record. Examination of this radiograph demonstrated the presence of the root canal treatment in the upper left lateral incisor. The corresponding post-mortem radiograph demonstrated the morphological similarities between the features common to both images.

While the evident similarities of the two images are sufficient to support the opinion that they derive from the same person, the differences in tube position and radiographic sensor from one image to the other do not permit direct comparison of the two images by superimposition.

\section{Case 2}

\section{Figure 2}


The missing person radiograph is on the left, and the image of the deceased is on the right. During a traumatic incident leading to death, the deceased had suffered severe fractures to both mandible and maxilla, and it was felt that post-mortem manipulation of the jaws would further damage the teeth. The supplied dental record contained a number of radiographs including the one shown above (left). A corresponding radiograph of the deceased was taken without disturbing the teeth or jaws, and the comparison is shown above.

Post-mortem access to this deceased person was restricted by the need to preserve evidence, and therefore the position of the x-ray tube and film sensor used in the patient radiograph could not be perfectly reproduced, with the result that there are visual differences in the restorations between the two images that require careful interpretation before an opinion can be formed. In this case, visual comparison of the constellation of individual features of the two images, including the features of the endodontic restoration, radicular and bony anatomy and the coronal restorations, suffices to suggest that they derive from the same person, but this cannot be demonstrated in a rigorous way; it remains an opinion based on careful interpretation and could be disputed in a courtroom. Fortunately, further ante-mortem radiographs of other dental quadrants were available and provided rigorous confirmation of the opinion (not shown).

This case clearly illustrates the difficulty in comparing radiographic images that have been taken where the $\mathrm{x}$-ray tube and the radiographic sensor have been placed in different positions, resulting in images that appear to have consequent differences. This underlines the importance of duplicating the position of both x-ray tube and radiographic sensor in order to duplicate as closely as possible the positional parameters between the ante-mortem and post-mortem images before comparison is undertaken. When one routinely compares radiographs of the same objects taken under different conditions, it becomes apparent how frequently the same things can look quite different on different images.

It also underlines the potential pitfalls that may arise in comparing a post-mortem radiograph with a written patient record, rather than with an ante-mortem radiograph, which is often undertaken if ante-mortem radiographs are not available. In a case of this type, we prefer to indicate that the features of the comparison are consistent with the ante-mortem records deriving from the same person (identity consistent), rather than a recommendation that they do derive from the same person (identity established).

The more successful duplication of ante-mortem and post-mortem radiographic parameters permits use of the preferred and more rigorous comparison techniques illustrated in cases three and four, below.

\section{Case 3}

\section{Figure 3}


The deceased person was severely putrefied and was not visually identifiable. A written dental record had been supplied by police for a missing person, and comparison of this record with the dental features of the deceased demonstrated complete consistency. A periapical radiograph (Figure 3a) was also supplied with the dental record, and it showed the presence of a completed root canal treatment in tooth 16. The corresponding radiograph of the deceased (Figure 3b) showed a root canal treatment in tooth 16 with features sufficient to demonstrate that the two images originated from the same individual, providing objective confirmation of identity. This similarity of features could be further demonstrated by image superimposition, showing the level of certainty in the evidence on which the expert opinion provided to the coroner was based (Figure 4).

\section{Figure 4}

\section{Case 4}

\section{Figure 5}

In subtraction image comparison, the ante-mortem radiograph and the post-mortem radiograph are superimposed. The upper of the two layers in the image is converted to a negative, and then its opacity is reduced until the common features of both images cancel out. In the event of an absolute correspondence, the result is a perfect neutral grey image. In real-world situations, however, there will rarely be perfect correspondence between two images taken at different times in different circumstances with different equipment, but there should be sufficient cancellation of common features to remove doubt about the similarity. Such a situation is demonstrated in Figure 5, indicating that the ante-mortem image and the post-mortem image derive from the same individual.

\section{Discussion}

Following a police investigation in the matter of a routine forensic identification, a particular missing person will have been tentatively identified as the most likely candidate to be a given deceased person. The forensic odontologist is then asked to compare any available dental records of that missing person with the dental features of the deceased person to determine if they are both likely to have originated from the same individual.

Thus, the dental features of the deceased are not being compared with the teeth of all other people on earth; this is a one-to-one comparison, and the possible outcomes are:

a) Identity Established: all dental features of both records exhibit complete concordance with no unexplained discrepancies on an objective basis such as image comparison. Identity can therefore be established beyond doubt;

b) Identity Consistent: all dental records exhibit concordance with no unexplained discrepancies, but insufficient numbers of features of sufficient probative value are present for comparison to determine identity beyond possible doubt. In Queensland, we also use this category if only written 
records are available without some feature or features of outstanding probative value that would elevate the case to (a) on the basis of their collective rarity;

c) Identity Possible: Identification cannot be excluded on the basis of the available information, but no stronger finding is possible. The value of this finding is that it does not close off the option for securing further dental records by subsequent police investigation or the use of other methods of identification in the same way that an exclusion does;

d) Insufficient Information Available: not enough information exists on which an opinion can be based. Again, this does not preclude the obtaining of further dental records or the use of different identification methods; and

e) Exclusion: The two sets of dental records clearly originate from different individuals. No further investigation into the presumed missing person is required for comparison to this deceased person, and it is clear that further investigation is required to determine their identity.

In Queensland, we will not indicate that identity has been established without comparing images, unless extraordinary evidence exists on which a stronger opinion can be given, as we feel that, in general, most written dental records cannot be demonstrated to provide the degree of rigour needed to assure the reliability of the outcome.

In the ideal case, post-mortem radiographs are taken in such a way that the original conditions under which the ante-mortem image was taken are duplicated as closely as possible. In such circumstances, similarity between the two images can be demonstrated by superimposition and, in an excellent case, digital subtraction of features common to both images.

While such comparisons are of great value, root canal treatments add an additional dimension of individuation to the process. The individual pulp chamber anatomy of a tooth can be described by its coronal pulp chamber morphology, and the number and location of canals, canal lengths and canal morphology. The number of root canals within a root canal system is not always consistent; the mesiobuccal root of maxillary molars will usually have a second canal $(2,3)$, the mandibular central incisor may have two root canals (4), the mandibular premolars may have multiple canals (5) as may the mandibular molars (6) and much variability exists in the root canal morphology of the maxillary premolars (7). In addition, variability in root canal lengths, curvatures, and other root canal configurations such as the C-Shaped canals (8), add a plethora of features which may distinguish any given root canal system from all others. An endodontically-treated tooth potentially contains more individuating information than a non-endodontically treated tooth, and as a result is a richer source of comparative image data.

The basic root filling consists of a cement sealer and a core filling material, most commonly gutta-percha. Other root fillings are silver points and most recently resinbased core filling materials. Zinc-Oxide Eugenol, resin, glass ionomer, silicone and calcium hydroxide are group classifications for endodontic sealers (9). The obturation of root canals, and hence post-preparation anatomy, will be demonstrated by the radio-opacity of these materials in a post treatment radiograph. 
Teeth that require endodontic treatment often also have substantial loss of coronal tooth structure henceforth also require complex restorations. Due to the loss of tooth structure associated with pathology or endodontic treatment, posterior teeth are commonly indicated for cusp coverage restorations $(10,11)$. Endodontic posts may be indicated in some circumstances. These posts may be active or passive, tapered or parallel, and prefabricated or custom cast. Nickel-Chromium alloy, stainless steel, titanium alloy, ceramic, zirconium and carbon fibre are materials commonly used in post fabrication (12). The complexity of the coronal restorations and variability in post and core material, design and placement provide further individuating features to each such treated tooth.

Endodontic procedures will commonly necessitate radiographs for diagnosis, treatment and evaluation of post-treatment success. The most useful of these for forensic purposes are the post-treatment radiographs, and these are of particular value in that endodontic restorations are less likely to be retreated or augmented than intracoronal restorations. Good radiographic technique produces radiographs which visualize individual root canals and reduce the superimposition of anatomical structures allowing a significantly improved image against which to evaluate or compare a post-mortem root canal radiograph.

Achieving a good post-mortem reproduction of a supplied radiograph provides a strong case for expressing an opinion confirming identity on the basis of a constellation of unique features, particularly if this can be achieved in more than one dental quadrant on the same individual. However, comparison of dental anatomical features in the absence of dental restorations or evidence of dental treatment is more complex than when such evidence is present. The multiple individuating features of less common and more complex treatments such as endodontic restorations provides an excellent basis for comparison.

We therefore hope for excellent post-treatment radiographs against which similar post-mortem radiographs can be compared to increase the evidence base on which an expert opinion can be provided.

We now routinely secure CT scans of post-mortem remains at QHFSS. With the increasing penetration of cone-beam technology into specialist and general dental practice, it is a matter of time before we are able to begin the comparison of threedimensional images, which will raise the degree of certainty still further.

\section{Conclusion}

We conclude that root canal treatments provide a wealth of morphological detail, providing rich data for the comparison of radiographs from a known missing person and an unknown deceased person, to answer the question of whether the two images are derived from the same person. A positive answer to this question permits the objective identification of the unknown deceased person as being the missing individual, and may add substantially to a case such as case 1 , where there is very little other available data.

\section{References:}


1. Pretty IA, Sweet D. A look at forensic dentistry - part 1: the role of teeth in the determination of human identity. Brit Dent J 2001; 190: 359-66.

2. Eskoz N, Weine FS. Canal configuration of the mesiobuccal root of the maxillary second molar. J Endod 1995;21:38-42.

3. Stropko JJ. Canal morphology of maxillary molars: clinical observations of canal configurations. J Endod 1999; 25: 446-50.

4. Benjamin KA, Dowson J. Incidence of two root canals in human mandibular incisor teeth. Oral Surg Oral Med Oral Pathol 1974; 38: 122-6.

5. Zillich R, Dowson J. Root canal morphology of mandibular first and second premolars. Oral Surg Oral Med Oral Pathol 1973; 36: 738-44.

6. Skidmore AE, Bjorndal AM. Root canal morphology of the human mandibular first molar. Oral Surg Oral Med Oral Pathol 1971; 32: 778-84.

7. Vertucci FJ, Seelig A, Gillis R. Root canal morphology of the human maxillary second premolar. Oral Surg Oral Med Oral Pathol 1974; 38: 45664.

8. Fan B, Cheung GSP, Fan M, Gutmann JL, Bian Z. C-shaped canal system in mandibular second molars: part I—anatomical features. J Endod 2004; 30: 899-903.

9. Orstavik D. Materials used for root canal obturation: technical, biological and clinical testing. Endo Topics 2005; 12: 25-38.

10. Aquilino SA, Caplan DJ. Relationship between crown placement and the survival of endodontically treated teeth. J Prosthet Dent 2002; 87: 256-63.

11. Nagasiri R, Chitmongkolsuk S. Long-term survival of endodontically treated molars without crown coverage: a retrospective cohort study. J Prosthet Dent 2005; 93: 164-70.

12. Schwartz RS, Robbins JW. Post placement and restoration of endodontically treated teeth: a literature review. J Endod 2004; 30: 289-301.

\section{Acknowledgements}

The authors wish to thank QHFSS and the Queensland State Coroner (Mr Michael Barnes) for kindly permitting them to reproduce material from selected de-identified coronial cases for the purposes of this paper.

\section{Figure Legends}

\section{Figure 1:}

(a) Periapical radiograph of Missing Person

(b) Corresponding periapical radiograph of Deceased

Figure 1

Figure 2:

(a) Periapical radiograph of Missing Person

(b) Corresponding periapical radiograph of Deceased

\section{Figure 3:}


(a) Periapical radiograph of Missing Person (b) Corresponding periapical radiograph of Deceased

Figure 3

Figure 4:
(a) 100\% Opacity
(b) $80 \%$ Opacity
(c) $60 \%$ Opacity
(d) $40 \%$ Opacity
(e) $20 \%$ Opacity
(f) $0 \%$ Opacity

Superimposition of radiograph of Deceased Person over supplied radiograph of Missing Person. Opacity of the post-mortem radiograph is progressively decreased, showing the close correlation between features including the endodontic restoration.

Figure 4

Figure 5:

$\begin{array}{lll}\text { (a) Ante-mortem radiograph } & \text { (b) Post-mortem radiograph } & \text { (c) Subtraction imaging }\end{array}$

Figure 5 


\section{Figures to show how images are laid out:}

(a)

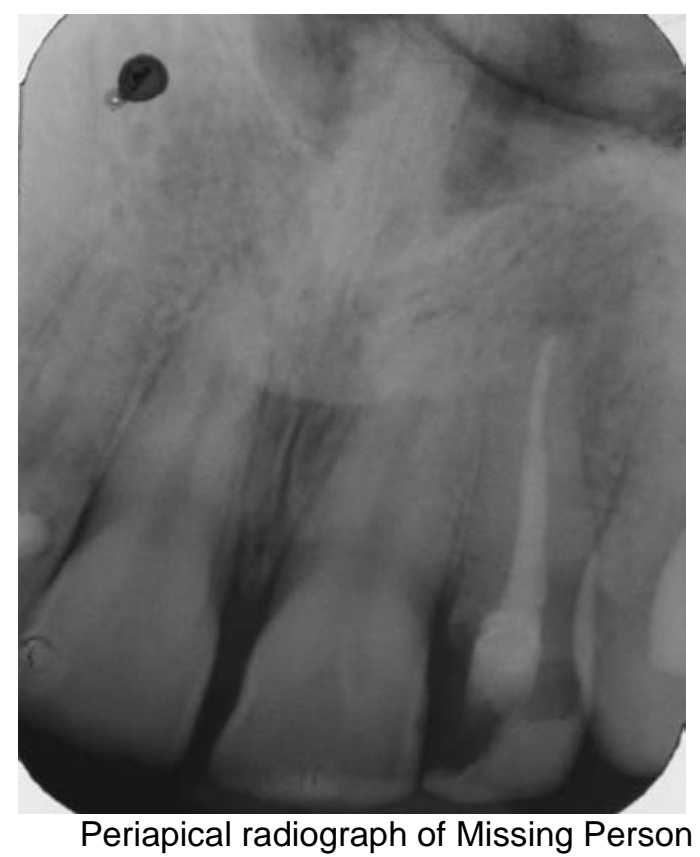

Figure 1

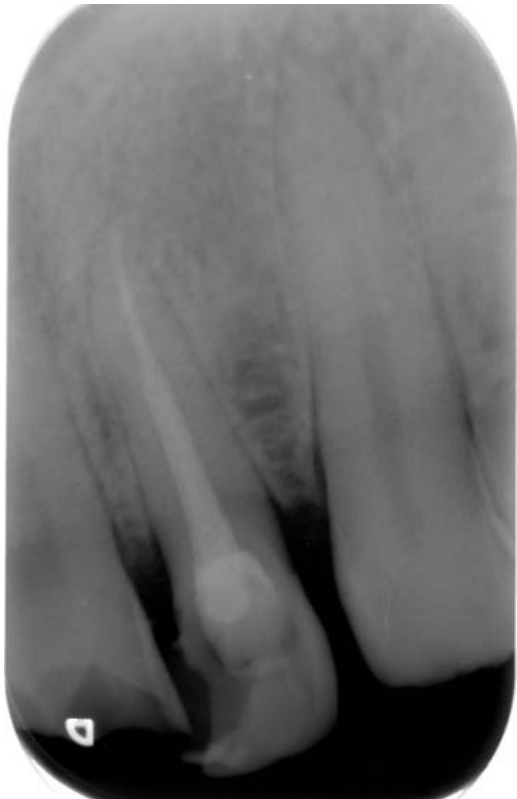

(b) Corresponding periapical radiograph of Deceased

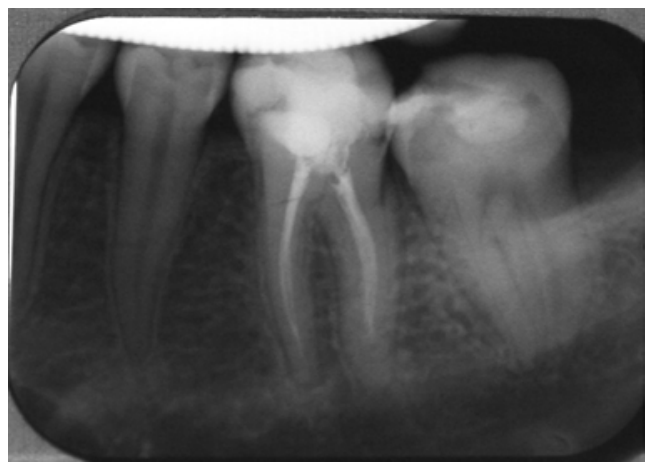

(a) Periapical radiograph of Missing Person

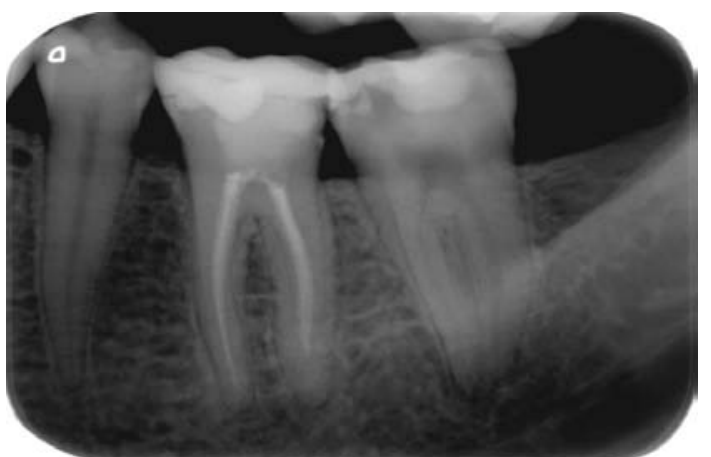

(b) Corresponding periapical radiograph of Deceased

Figure 2

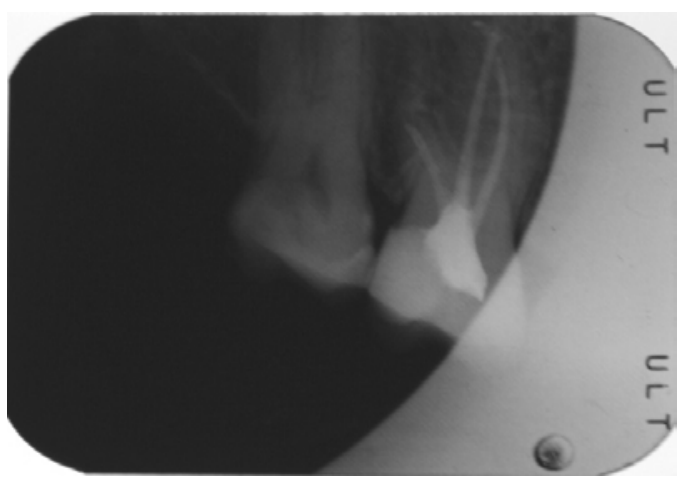

(b) Periapical radiograph of Missing Person

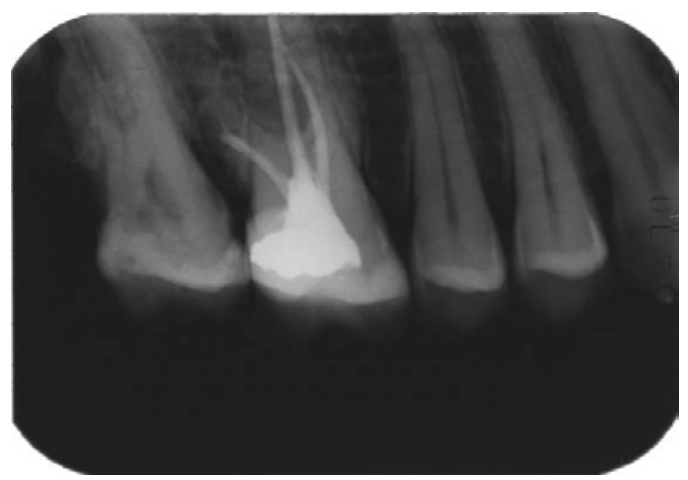

(b) Corresponding periapical radiograph of Deceased

Figure 3 


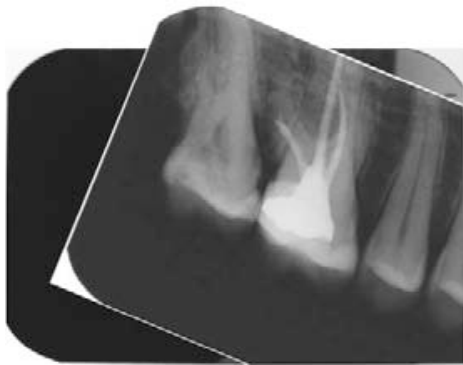

(a) $100 \%$ Opacity

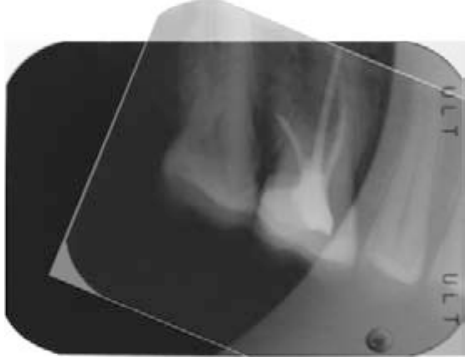

(d) $40 \%$ Opacity

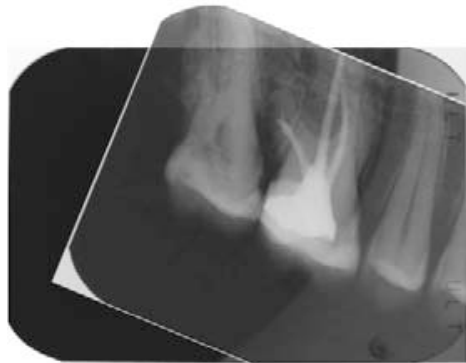

(b) $80 \%$ Opacity

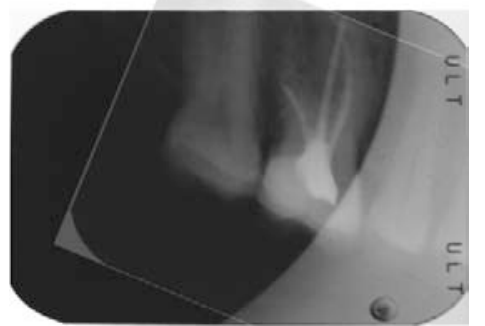

(e) $20 \%$ Opacity

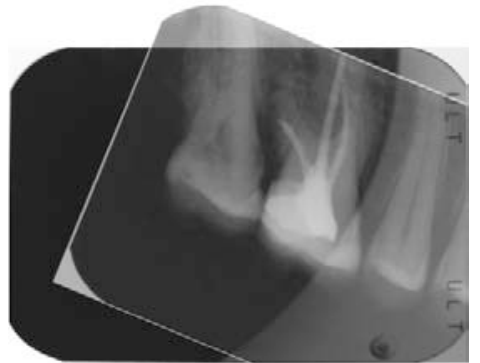

(c) $60 \%$ Opacity

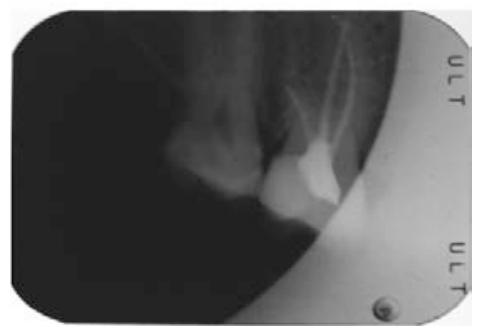

(f) $0 \%$ Opacity

Superimposition of radiograph of Deceased Person over supplied radiograph of Missing Person. Opacity of the post-mortem radiograph is progressively decreased, showing the close correlation between features including the endodontic restoration.

Figure 4

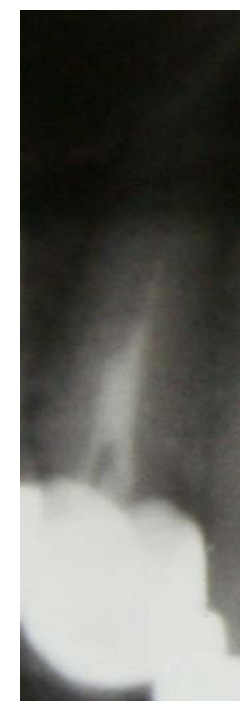

(a) Ante-mortem radiograph

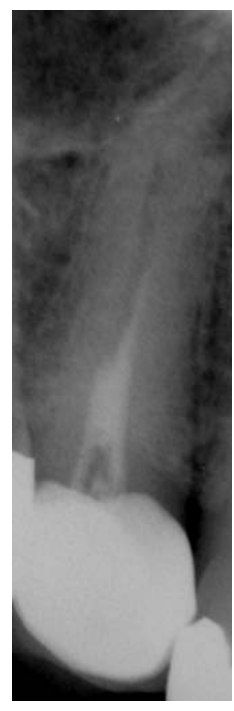

(b) Post-mortem radiograph

Figure 5

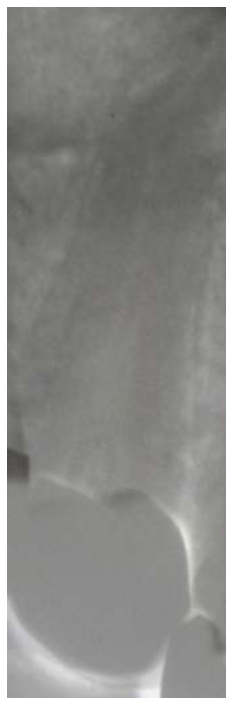

(c) Subtraction imaging 